\title{
Heavy-Electron Metals: An Exciting Frontier in Quantum Matter
}

\author{
DOI: $10.3938 /$ PhiT. 23.010
}

\section{J. D. Thompson}

Heavy-electron metals, with an effective mass of itinerant charge carriers approaching 1000 times the mass of a free electron, exemplify the consequences of strong electronic correlations. One important consequence is the emergence of unconventional Cooper pairing of heavy electrons as these materials are tuned to a magnetic quantum-critical state. The continued study of heavy-electron metals provides an opportunity to develop an appropriate theory of magnetic quantum criticality in correlated metals and of how associated fluctuations induce Cooper pairing.

When a dilute number of magnetic ions is incorporated into a metallic host, the antiferromagnetic interaction of the magnetic ion's localized spin with the spin of the host's conduction electrons can produce a resonance in the electronic density of states. In the limit of a single magnetic ion, this many-body problem, the Kondo-impurity problem, has been solved exactly by renormalization group ${ }^{[1]}$ and Bethe ansat ${ }^{[2]}$ techniques. A consequence of the resonance is a huge electronic contribution to the specific heat, giving a Sommerfeld coefficient of specific heat $\gamma$ per mole of localized spins that scales inversely with the Kondo temperature. That is, $\gamma \propto 1 /$ $T_{\mathrm{K}}$, where $T_{\mathrm{K}} \sim E_{\mathrm{F}} \exp [-1 / 2 J N(0)]$, with $E_{\mathrm{F}}$ the Fermi energy, $J$ the exchange between localized and itinerant spins and $N(0)$ the unrenormalized density of states of the conduction band. At temperatures $T \ll T_{\mathrm{K}}$, the spins of conduction electrons collectively compensate the localized spin, creating a net spin-singlet Fermi liquid and, from free electron relationships, the associated huge Sommerfeld co-

\section{저자약력}

Joe D. Thompson obtained a Ph. D. from the University of Cincinnati (1975). He was postdoctoral scientist (1975-1977), staff member (1977-2001), and group leader (1992-2001) at Los Alamos National Laboratory and has been a Laboratory Fellow since 2001. His research has focused on discovering new physics through new materials, with an emphasis on the behavior of correlated electron materials at low temperatures, high pressures and high magnetic fields. (jdt@lanl.gov) efficient implies a correspondingly huge effective mass of itinerant quasiparticles.

The discovery of a very large $\gamma$ per mole $\mathrm{Ce}$ in $\mathrm{CeAl}_{3}$, with a periodic array of $\sim 10^{23}$ localized Ce $4 \mathrm{f}$-electron spins, solicited an analogy to the Kondo-impurity problem. But, a direct analogy was recognized as inappropriate because scattering by a Kondo impurity produces a large residual resistivity/impurity. Though the resistivity of $\mathrm{CeAl}_{3}$ does increase with decreasing temperature, like a collection of non-interacting Kondo impurities, it passes over a maximum near $30 \mathrm{~K}$ and drops to a very small value in the limit that temperature approached zero. ${ }^{[3]} \mathrm{CeAl}_{3}$ and subsequently discovered Ce-, $\mathrm{Yb}$ - and U-based metallic compounds with properties like those of $\mathrm{CeAl}_{3}$ are called heavy-electron (or more generally, heavy-fermion) materials because the effective mass of their itinerant charge carriers approaches 1000 times the mass of a free electron. ${ }^{[4]}$ Unlike the impurity limit, lattice periodicity of localized or nearly localized spins in heavy-fermion materials has two important consequences: one is that periodicity requires a band description of the heavy quasiparticles at very low temperature, and the other is that these spins couple through the indirect Ruderman-KittelKasuya-Yosida (RKKY) interaction that depends on $J^{2} N(0)$. A microscopic theory of how a dense lattice of non-interacting 'Kondo impurities' at room temperature evolves with decreasing temperature to become a band of itinerant heavy quasiparticles remains an extremely challenging problem for many-body physics. Recent advances in Dynamical Mean Field Theory techniques have begun to capture aspects of the

\section{REFERENCES}

[1] K. G. Wilson, Rev. Mod. Phys. 47, 773 (1975).

[2] N. Andrei, K. Furuya and J. H. Lowenstein, Rev. Mod. Phys. 55, 331 (1983).

[3] K. Andres, J. E. Gaebner and H. R. Ott, Phys. Rev. Lett. 35, 1779 (1975).

[4] For example, Z. Fisk et al., Science 239, 33 (1988).

[5] J. H. Shim, K. Haule and G. Kotliar, Science 318, 1615 (2007). 


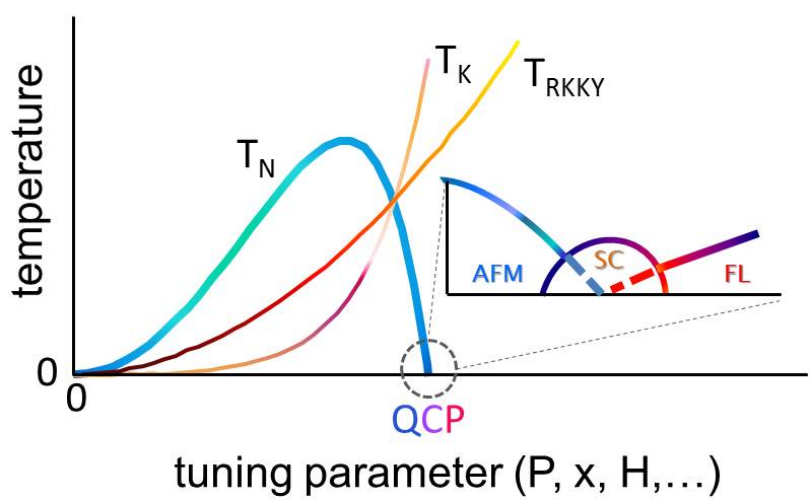

Fig. 1. Schematic illustration of the competition between Kondo $\left(T_{K}\right)$ and RKKY (T RKKY $)$ interactions, considered originally by S. Doniach. ${ }^{[6]}$ This competition leads to a non-monotonic variation of the Néel temperature $\left(T_{N}\right)$ as a function of some non-thermal tuning parameter, such as pressure $(\mathrm{P})$, chemical composition $(\mathrm{x})$ or magnetic field $(\mathrm{H})$. These tuning parameters tend to change the magnetic exchange $J$ upon which both $T_{K}$ and $T_{\text {RKKY }}$ depend. At a critical value of the tuning parameter, the Néel boundary reaches zero temperature, a magnetic quantum critical point (QCP). An expanded view around the QCP shows a dome of superconductivity (SC) and the emergence of a Landau Fermi-liquid (FL) state. Normal state properties of materials around the QCP are distinctly different from expectations of a Landau Fermi-liquid.

physics. ${ }^{[5]}$ Extending these techniques to clusters and to lower temperatures holds promise for future progress in this area.

Because of the RKKY interaction, several of these materials order magnetically before the heavy-fermion state fully develops, but the balance between the competing tendencies to order magnetically (the RKKY interaction) and to form a non-magnetic state (through the Kondo effect) can be tuned easily by chemistry or pressure (See Fig. 1). Chemistry can change the electronic density of states $N(0)$ and spatial separation of the localized spins, upon which the magnitude and sign of $J$ depend. Though very useful, chemistry has the drawback that it introduces undesirable crystalline disorder, in contrast to hydrostatic pressure which is a 'clean' variable that does not add disorder. The relatively weak exchange interaction in these systems is a strong function of volume, and because both the RKKY and Kondo interaction depend on $J$, but with different functional forms, modest pressures often are sufficient to change the ground state dramatically. Indeed, a number of experiments have shown that pressures of order one to a few GPa drive the electronic system from a magnetically ordered to a heavy-fermion paramagnetic ground state. CeIn $n_{3}$ is a typical example. ${ }^{[7]}$ It orders antiferromagnetically at $10 \mathrm{~K}$ under atmospheric pressure, and a pressure of $2.5 \mathrm{GPa}$ tunes its Néel temperature to zero, that is, to a magnetic quantum-critical point.
What was most surprising in these experiments on $\mathrm{CeIn}_{3}$ was that as its $T=0$ magnetic/non-magnetic boundary was approached, a narrow dome of superconductivity emerged that was centered on the critical pressure..$^{[7]}$ This discovery led to the suggestion that magnetic fluctuations might provide an attractive interaction that pairs superconducting electrons. Even before this discovery, there was a precedent for this suggestion from earlier studies of the ambient-pressure heavy-fermion superconductors $\mathrm{CeCu}_{2} \mathrm{Si}_{2}, \mathrm{UPt}_{3}$ and $\mathrm{UBe}_{13}{ }^{[8]}$ In each of these cases, Cooper pairing of heavy quasiparticles developed out of a strongly paramagnetic normal state, which is antithetical to conventional, phonon-mediated s-wave superconductivity, and slight changes in chemistry induced magnetic order in them. Experimental and theoretical studies of these and subsequently discovered ambient-pressure heavy-fermion superconductors have found compelling evidence that the symmetry of Cooper pairs breaks lattice symmetry, a hallmark of unconventional superconductivity. ${ }^{[9]}$ Though it seems very likely, there still is no proof that magnetic fluctuations mediate Cooper pairing, and this is an important task for experiment. Solving this problem in heavy-fermion superconductors will provide a foundation for understanding the role that magnetism also may play in producing superconductivity in other correlated electron systems, such as the cuprates and iron-pnictides. ${ }^{[10]} \mathrm{A}$ microscopic theory of magnetically mediated superconductivity is lacking but would be valuable benchmark for experiment.

An important question raised by these discoveries is the relationship between magnetic and superconducting electrons. In conventional superconductors, magnetic electrons break time-reversal symmetry of Cooper pairs: consequently, magnetic and superconducting electrons should be distinct. The study of heavy-fermion systems in which unconventional superconductivity and magnetism appear in the same material have established that magnetism and superconductivity coexist microscopically in real space and that both orders in-

\section{REFERENCES}

[6] S. Donaich, Physica B 91, 231 (1977).

[7] N. D. Mathur et al., Nature 394, 39 (1998).

[8] For example, K. Miyake, S. Schmitt-Rink and C. M. Varma, Phys. Rev. B 34, 6554 (1986); D. J. Scalapino, E. Loh and J. E. Hirsch, Phys. Rev. B 34, 8190 (1986).

[9] For example, M. Sigrist and K. Ueda, Rev. Mod. Phys. 63 , 239 (1991).

[10] D. J. Scalapino, Rev. Mod. Phys. 84, 1383 (2012). 
volve the same electrons. ${ }^{[1]}$ One interpretation of these observations is that both orders arise from an instability of the Fermi surface, i.e., compete in momentum space; however, an alternative interesting possibility is that the f-electron adopts a dual role, simultaneously having itinerant and localized characters. How these interpretations might be related and whether a single interpretation might be applicable to all cases are open questions.

In the limit of zero temperature, the normal state of some heavy-fermion systems, eg., $\mathrm{CeAl}_{3}$ and $\mathrm{UPt}_{3}$, have characteristics of a Landau Fermi liquid - a constant Sommerfeld coefficient, a uniform magnetic susceptibility $\chi$ related to $\gamma$ by the Wilson ratio, and a resistivity $\rho$ that increases as $T^{2}$ with the notable property that all are enhanced by a factor proportional to the effective mass of heavy quasiparticles. ${ }^{[4]}$ There are, however, a growing number of examples, including almost all heavy-fermion superconductors, that exhibit properties distinctly different from expectations of a Landau Fermi liquid. ${ }^{[12]}$ In some cases, these non-Fermi liquid properties appear to result from disorder and can be described approximately in terms of Griffiths phase concepts or as a result of a distribution of Kondo temperatures. On the other hand, non-Fermi liquid properties in nearly disorder-free materials require a different interpretation. ${ }^{[13]}$

Excitations from a magnetic quantum-critical point are known to induce strongly non-Fermi-liquid properties, and indeed, these 'clean' materials are at or close to a zero-temperature magnetic/non-magnetic boundary. If the nearby magnetic order is of a spin-density wave type, a quantum generalization of the theory of critical fluctuations at a classical phase transition accounts for some properties of some non-Fermi liquid materials. The lack of complete agreement between this theory and experimental results on several materials has motivated new proposals for quantum criticality, such as a breakdown of the Kondo effect, fractionalization of heavy quasiparticles into a chargeless spin object and a spinless fermion, as well as other ideas. ${ }^{[13]}$ Presently, there is no consensus for a theory of quantum criticality in heavy-fermion systems. This is a significant issue because there is growing evidence that critical fluctuations emerging from a quantum-critical point may be important for superconductivity. ${ }^{[14]}$ Irrespective of this problem, heavy-fermion materials are ideal candidates for studying quan-

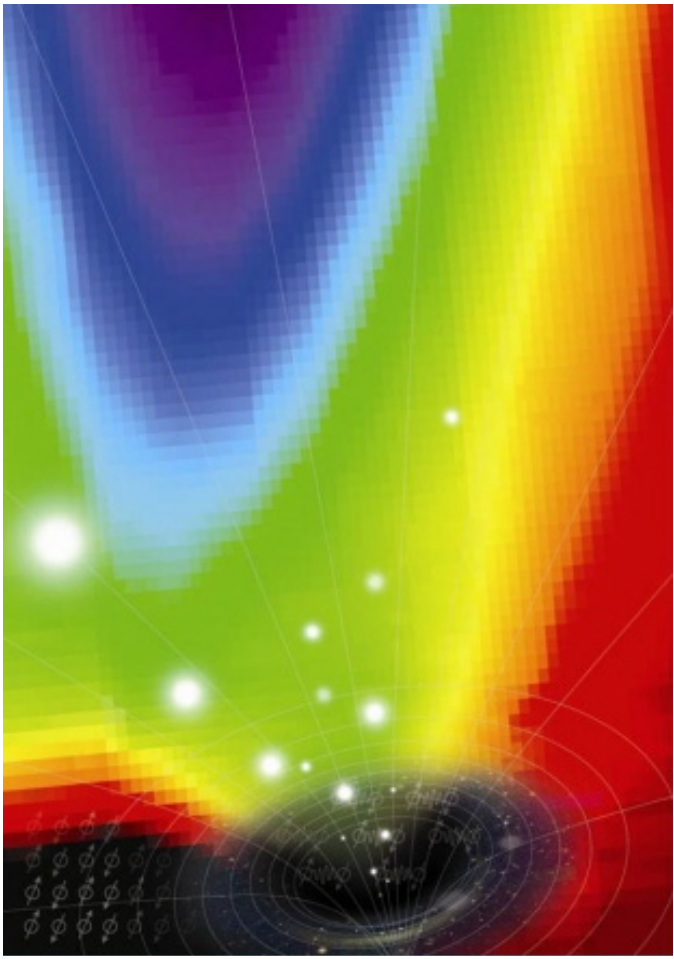

Fig. 2. Artist's suggestion of the connection between the physics of a black hole and the physics of magnetic quantum criticality in heavy-fermion materials.

tum-critical phenomena, which are found in ever increasing classes of materials. A fascinating twist to this problem has been the recent realization that quantum criticality in heavy-fermions may be cast in the mathematical framework of dynamics near the horizon of a black hole, Fig. 2. ${ }^{[15]}$ If this idea withstands future tests, it opens the possibility of exploring the physics of the cosmos through bench-top experiments on quantum critical heavy-fermion materials.

Heavy fermions have proven to be fertile ground from which new concepts and new challenges have grown. As prototypes of strongly correlated electron physics and of new states of matter that can emerge from electronic correlations, these systems define an exciting frontier in condensed matter physics that is certain to continue to inform and surprise us.

\section{REFERENCES}

[11] For example, T. Park et al., Proc. Natl. Acad. Sci. USA 105, 6825 (2008).

[12] For example, Q. Si and F. Steglich, Science 329, 1161 (2010).

[13] H. v. Löhneysen, A. Rosch, M. Vojta and P. Wölfle, Rev. Mod. Phys. 79, 105 (2007).

[14] O. Stockert et al., Nature Phys. 7, 119 (2011).

[15] For example, S. Sachdev and B. Keimer, Phys. Today 64, 29 (2011). 